\title{
Mediastinal Granulocytic Sarcoma
}

National Cancer Institute

\section{Source}

National Cancer Institute. Mediastinal Granulocytic Sarcoma. NCI Thesaurus. Code C146883.

A malignant tumor that arises from the mediastinum and is composed of myeloblasts, neutrophils and neutrophil precursors. It is the most common type of myeloid sarcoma affecting the mediastinum. 